\title{
Kalman Filter-Based Architecture for Robust and High-Sensitivity Tracking in GNSS Receivers
}

\author{
José A. Del Peral-Rosado*, José A. López-Salcedo*, Gonzalo Seco-Granados*, \\ José M. López-Almansa ${ }^{\dagger}$, Joaquín Cosmen ${ }^{\dagger}$ \\ *Signal Processing for Communication and Navigation Group (SPCOMNAV), \\ Universitat Autònoma de Barcelona, 08193 Bellaterra (Barcelona), Spain \\ ${ }^{\dagger}$ GMV, Isaac Newton, 11, PTM Tres Cantos, 28760 Madrid, Spain
}

\begin{abstract}
Blockage of the line-of-sight (LOS) component is one of the main problems of GNSS receivers when operating in harsh working conditions. This is the case, for instance, of urban canyons, where GNSS receivers in moving vehicles are subject to sudden fading events with more than $20 \mathrm{~dB}$ of signal attenuation. In these circumstances it is not possible for traditional receivers to keep track of the received signals, thus failing to provide reliable position fixes. The problem is aggravated when hardware implementation constraints need to be satisfied. For instance, as in target applications that involve embedding GNSS capabilities into ARM processors of mobile devices. Therefore, highsensitivity GNSS receivers with reduced complexity and power consumption are mandatory. The contribution of this paper is the proposal and analysis with synthetic signal of a Kalman filterbased architecture for robust and high-sensitivity tracking. The use of extended correlations mitigates the measurement noise and allows the tracking operation under these harsh conditions. The results obtained validate the strategy proposed to operate at a $C / N_{0}$ (carrier-to-noise ratio) as low as $15 \mathrm{dBHz}$.
\end{abstract}

\section{INTRODUCTION}

During the recent years, the use of Global Navigation Satellite Systems (GNSS) has extraordinarily increased and extended to many areas. New applications and services have been created, and GNSS receivers have become more advanced and efficient, leading to their incorporation even into small and portable devices such as mobile phones. Such an unprecedented development has been mainly motivated by the issue of legal mandates for the location identification of emergency calls. These mandates (the FCC E911 in the US and the E112 recommendation in Europe) demand mobile terminals to be able to report their position with accuracies below 50 and $150 \mathrm{~m}$, for $67 \%$ and $95 \%$ of the time, respectively.

Without any doubt, these requirements have emerged as a catalyst for the research and development in GNSS receiver technology, and the motivation to face new challenges such as the operation of GNSS receivers in harsh environments. With mobile terminals being operated either outdoors or indoors, in sparsely populated areas or in deep urban scenarios, a

The work of José A. Del Peral-Rosado has been funded by GMV Aerospace and Defence in the framework of an educational collaboration program with the Degree of Master of Science in Design of Telecommunication Systems at UAB. SPCOMNAV is partially funded by the Spanish Government under projects TEC2008-06305, the Catalan Government under grant 2009 SGR 298, and the Chair of Knowledge and Technology Transfer UAB Research Park - Santander. The authors are also affiliated with IEEC-UAB. myriad of possible working conditions may actually arise. The common feature of all these new working scenarios is the presence of blocking obstacles and propagation disturbances that prevent GNSS receivers from observing the expected perfect clear-sky conditions that were assumed in the nominal design of the system.

In view of these evidences, the motivation of the present work is to improve the operation of GNSS receivers in urban areas by providing the means to track the received signal despite of being affected by signal fading or blockage. Receivers operating under these working conditions are often referred to as high-sensitivity (HS) GNSS receivers, and the fact of keeping track of the received signal, as signal tracking. Thus, the objective to be pursued here involves the design of improved high-sensitivity tracking algorithms, with special emphasis in their application to urban areas and the incorporation into moving vehicles.

Moreover, in order to be incorporated in mass-market vehicles, the cost of the GNSS receiver is also an important issue to be kept in mind. If the receiver (due to the required HS techniques) is too expensive, vehicle manufacturers will be reluctant to incorporate it or will even not consider its adoption, in favour of other less expensive technological solutions. Hardware simplicity is thus one of the goals to be sought in the present work, with the idea of being able to implement the proposed HS algorithms in low-cost and reduced complexity ARM processors.

Therefore, a computationally efficient architecture able to operate under high-sensitivity conditions is required. For these purposes, Kalman filter-based tracking appears to be the most suitable solution. In the literature, there are many studies that have included Kalman filter-based approaches into their architectures. For instance, in [1], [2], the tracking architecture is based on an extended Kalman filter (EKF) that substitutes the discriminator and the loop filter of the traditional tracking loops. Nevertheless, this architecture implies an extra processing load due to the computation of the Jacobian of the measurement function. In order to circumvent this drawback, a linear Kalman filter (KF) is proposed in [3], [4], which just substitutes the traditional loop filter using the discriminator outputs as measurements. The robustness of the KF with respect to the conventional tracking loops is demonstrated 
in [4], obtaining accuracy improvements in the carrier and Doppler tracking and better position performance in harsh conditions.

In this paper, the linear Kalman filter-based tracking is presented as the base architecture for implementing the HSGNSS receiver. However, it may be very difficult, if not impossible, for a conventional Kalman filter to track signals in weak signal environments. Thus, some signal processing improvements are proposed by introducing the concept of extended correlations, where several $1 \mathrm{~ms}$-correlation outputs are combined, resulting in a high-sensitivity discriminator. Once this HS discriminator is incorporated into the Kalman architecture, the performance of the proposed tracking structure is evaluated. Lastly, conclusions and future work are discussed.

\section{Signal Model}

The received signal at the antenna output contains the contributions of $L$ satellites in view corrupted by additive white Gaussian noise $\eta_{R F}(t)$ with spectral density (PSD) $N_{0} / 2$ :

$$
r_{R F}(t)=\sum_{i=1}^{L} y_{R F, i}(t)+\eta_{R F}(t) .
$$

Each bandpass satellite signal $y_{R F, i}(t)$ can be written as

$$
y_{R F, i}(t)=A_{i} d_{i}\left(t-\tau_{i}\right) c_{i}\left(t-\tau_{i}\right) \cos \left(2 \pi f_{i} t+\phi_{i}\right),
$$

where $A_{i}$ is the signal amplitude, $d_{i}$ is the navigation message, $c_{i}$ is the spreading code, $\tau_{i}$ is the code delay, $f_{i}$ is the carrier frequency plus the carrier Doppler frequency shift $f_{D}$, and $\phi_{i}$ is the carrier phase.

The signal of (1) is downconverted, filtered, sampled and digitized for each satellite channel by the receiver front-end resulting in an intermediate frequency (IF) signal, denoted by $r\left(n T_{s}\right)$, where $T_{s}$ is the sampling period. Once the IF signal is obtained, it is correlated with a locally-generated replica of this incoming signal $\hat{y}_{i}\left(n T_{s}\right)$. The replica signal is generated according to some tentative code delay $\hat{\tau}$ and Doppler frequency $\hat{f}$. If the tentative values of $\hat{\tau}$ and $\hat{f}$ are close enough to the true ones, this operation produces the carrier and code wipe-off. Then, the correlated samples are accumulated over the integration time $T$ (in seconds), where $T$ is usually a C/A code epoch period (i.e. one $\mathrm{ms}$ for the case of GPS L1 signals). The integrations are stored and dumped, resulting in an integration-and-dump (I\&D) filtering of the signal components.

Since the correlated and integrated signal is obtained at time $t_{k}$, the index $k$ represents the iterative behaviour of the tracking architecture for one satellite channel, which results in the following output signal [5]

$$
Y_{k} \approx A_{k} d_{k} R\left(\tau_{e, k}\right) \frac{\sin \left(\pi f_{e, k} T\right)}{\pi f_{e, k} T} e^{j\left(\pi f_{e, k} T+\phi_{e, k}\right)}+\eta_{k},
$$

where $A_{k}$ is the signal amplitude, $d_{k}$ is the data bit, $R(\cdot)$ is the code correlation function, and $\left[\tau_{e}, \phi_{e}, f_{e}\right]$ denote the code delay error, carrier phase error, and Doppler shift error, respectively. Assuming the noise variance normalized to 1 , the I\&D signal amplitude can be defined as $A_{k} R(0)=\sqrt{\left(C / N_{0}\right)_{k} T}$. Moreover, the $R(\cdot)$ function can be ideally expressed (for a infinite-bandwidth correlation and ideal code) as

$$
R\left(\tau_{e}\right)= \begin{cases}1-\left|\tau_{e}\right| & \text { for } \tau_{e} \leq T_{\text {chip }}, \\ 0 & \text { otherwise }\end{cases}
$$

where $T_{\text {chip }}$ is the chip period.

As it can be noticed from (3), the complex argument of $Y_{k}$ represents the average carrier phase error over the integration interval $\pi f_{e, k} T+\phi_{e, k}$, instead of the $k$-th true carrier phase error at the end of the interval $2 \pi f_{e, k} T+\phi_{e, k}$. This averaging effect produces an offset error in the carrier estimation that increases with the frequency error $f_{e, k}$ or the integration time $T$. Nonetheless, this effect is ignored in traditional receivers because its impact is negligible for an integration of just one code period.

Lastly, the term $\frac{\sin \left(\pi f_{e, k} T\right)}{\pi f_{e, k} T}$ or $\operatorname{sinc}\left(f_{e, k} T\right)$ may also have an impact on the correlation. Actually, it acts as an attenuation factor. Nevertheless, the approximation $\sin (x) \approx x$ for $0 \leq x \leq 0.3$ can be fulfilled for usual values of the code periods $T$ in GNSS provided that the frequency error does not exceed about $[25-100] \mathrm{Hz}$, which is a considerable error at the tracking stage. Under these assumptions, this term can be approximated as $\operatorname{sinc}\left(f_{e, k} T\right) \approx 1$.

\section{Kalman Filter-BASED TRACKING}

\section{A. Motivation}

The Kalman filter-based tracking implements locally the KF (at every satellite channel), substituting the traditional tracking loops. In fact, the traditional tracking loops can be seen as a particular case of the Kalman filter with constant coefficients, as it is demonstrated in [6], [7]. Thus, the main advantage of the KF with respect to the traditional approach is that the loop filter bandwidth is optimally adjusted via the time-varying Kalman gains. This adaptive nature easily allows to track the signal under changing conditions. Therefore, the performance of the conventional tracking architecture can be improved by implementing a Kalman filter.

The KF uses a dynamical and a statistical model of the system to predict and correct the estimated parameters, being optimal in the mean square error (MSE) sense for Gaussian input data. Unlike conventional tracking, the estimated parameters are updated taking into account the measurement noise and the uncertainty in its current estimates, being robust for very noisy conditions. Moreover, this architecture may have the ability to bridge signal outages by propagating the current estimated parameters with its dynamic model. Another advantage of this structure is that the Kalman filterbased approach can be used as a previous step to implement vector tracking [8]-[10]. Finally, the receiver specifications can be fulfilled due to the following two reasons: its realtime application can be ensured due to its low computational burden, which is comparable to that of the traditional tracking; and, the extension of the integration time can be included in the architecture to achieve high sensitivity. 


\section{B. Architecture}

The goal of our tracking architecture is to directly estimate the signal parameters instead of the signal parameter errors obtained after the discriminator. Thus, the estimated parameters will be used directly to generate the replica signal $\hat{y}_{i}\left(n T_{s} ; \hat{\mathbf{x}}_{k}\right)$. This operation simplifies the implementation complexity, similarly to the option 4 introduced in [4]. This architecture can be interpreted as an extended Kalman filter (EKF) due to the non-linear measurement function $h\left(\hat{\mathbf{x}}_{k}\right)$, as is shown in Figure 1.

Despite of the EKF analogy, it should be remarked that the conversion from the estimated state vector $\hat{\mathbf{x}}_{k}$ to the replica signal $\hat{y}_{i}\left(n T_{s} ; \hat{\mathbf{x}}_{k}\right)$ is done by the code and carrier generator, which are already implemented in traditional tracking loops. Moreover, the combination of the correlation operation and the discriminators is equivalent to the difference between the current state vector $\mathbf{x}_{k}$ and the estimated $\hat{\mathbf{x}}_{k}$, thus resulting indeed in the natural procedure of the linear KF (i.e. the error signal is linear with the Kalman state vector). In the following sections, the implementation of this architecture is discussed.

\section{System dynamic model}

The system dynamic model is defined by

$$
\mathbf{x}_{k+1}=\mathbf{F} \mathbf{x}_{k}+\mathbf{G w}_{k} .
$$

At time $t_{k}$, the $(k+1)$-th state vector $\mathbf{x}_{k+1}$ is generated by projecting ahead the current state vector $\mathbf{x}_{k}$. This prediction is done by the transition matrix $\mathbf{F}$ and it allows the update of the signal parameters when no measurements are available (e.g. during extended correlation periods). Moreover, the colored noise transition matrix $\mathbf{G}$ and the state transition noise vector $\mathbf{w}_{k}$ can model the non-deterministic dynamic processes of the GNSS signal. The state transition noise vector $\mathbf{w}_{k}$ is assumed to be a zero-mean uncorrelated Gaussian noise process that satisfies:

$$
\begin{aligned}
E\left[\mathbf{w}_{k}\right] & =0, \\
E\left[\mathbf{w}_{k} \mathbf{w}_{n}^{H}\right] & =\mathbf{Q} \delta_{k n},
\end{aligned}
$$

where $\delta_{k n}$ denotes the Kronecker's delta, and $\mathbf{Q}$ is the state transition noise covariance matrix. The covariance matrix $\mathbf{Q}$ can be designed to model the non-deterministic dynamic processes, as it is done in [1], [3], [11]. However, in the current implementation, it is preferred to initially tune manually the matrix $\mathbf{Q}$.

Taking into account the signal model described in Section II, the system dynamic model is formulated based on the main parameters to estimate, which describe the incoming signal $r\left(n T_{s} ; \mathbf{x}_{k}\right)$ and are the code delay $\tau$ (in chips), the carrier phase $\phi$ (in cycles) and the carrier Doppler frequency shift $f_{D}$ (in Hz). Nevertheless, the carrier Doppler frequency rate $f_{D}$ (in $\mathrm{Hz} / \mathrm{s}$ ), which is the derivative of $f_{D}$, is also present due to the acceleration along the line-of-sight (LOS) between the satellite and the receiver. Therefore, the state vector is defined by the estimation of the above-mentioned parameters:

$$
\mathbf{x}_{k}=\left[\begin{array}{llll}
\tau & \phi & f_{D} & \dot{f}_{D}
\end{array}\right]_{k}^{T} .
$$

Since the measurements arrive each $T$ seconds, this time is considered outside and the system dynamic model is discretized in order to simplify its implementation, which yields

$$
\mathbf{F}=\left[\begin{array}{cccc}
1 & 0 & \beta & \frac{\beta}{2} \\
0 & 1 & 1 & \frac{1}{2} \\
0 & 0 & 1 & 1 \\
0 & 0 & 0 & 1
\end{array}\right]
$$

where $\beta$ is the carrier aiding factor defined by the relation between the C/A code chip rate $R_{c}$ and the carrier frequency [12]. In the current implementation, the states $f_{D}$ and $\dot{f_{D}}$ are normalized.

\section{Measurement model}

The measurement model is described as

$$
\mathbf{z}_{k}=\mathbf{H x}_{k}+\mathbf{v}_{k},
$$

where $\mathbf{z}_{k}$ is the measurement vector, $\mathbf{H}$ is the observation matrix and $\mathbf{v}_{k}$ is the measurement noise vector. The KF filter assumes that the measurement noise vector $\mathbf{v}_{k}$ is a zero-mean uncorrelated Gaussian noise process and independent to $\mathbf{w}_{k}$ :

$$
\begin{aligned}
E\left[\mathbf{v}_{k}\right] & =0, \\
E\left[\mathbf{v}_{k} \mathbf{v}_{n}^{H}\right] & =\mathbf{R}_{k} \delta_{k n}, \\
E\left[\mathbf{w}_{k} \mathbf{v}_{n}^{H}\right] & =0, \quad \text { for all } k \text { and } n,
\end{aligned}
$$

where $\delta_{k n}$ denotes the Kronecker's delta and $\mathbf{R}_{k}$ is the measurement noise covariance matrix. Since we focus on the linear Kalman filter, non-linear discriminators are needed to obtain error signals that are a linear function of the state vector. Basically, the correlation operation and the discriminator computes the difference between the states of the incoming and replica signal, and the output is the Kalman measurement errors. The implementation of this structure is based on the code delay error $\tau_{e, k}$ and the carrier phase error $\phi_{e, k}$, resulting in the measurement vector $\mathbf{z}_{k}=\left[\tau_{e}, \phi_{e}\right]_{k}^{T}$. Thus, the observation matrix can be deduced from the measurement equation (10), which results in the following expression:

$$
\mathbf{H}=\left[\begin{array}{llll}
1 & 0 & 0 & 0 \\
0 & 1 & 0 & 0
\end{array}\right] .
$$

The adopted discriminators have been selected according to their pull-in range, computational burden and optimality at low $C / N_{0}$. Focusing on the code or DLL discriminator, the popular normalized early minus late envelope discriminator is chosen, and it is defined by

$$
\tau_{e, k}=(1-\Delta) \frac{\left|Y_{E, k}\right|-\left|Y_{L, k}\right|}{\left|Y_{E, k}\right|+\left|Y_{L, k}\right|},
$$

where the subscripts $E$ and $L$ denote the early and late correlator outputs, respectively, and $\Delta$ is the early and late spacing with respect to the prompt correlator output, which is usually 0.5 chip.

In the absence of data bits, pure carrier or PLL discriminators can be used, improving the tracking threshold by up 


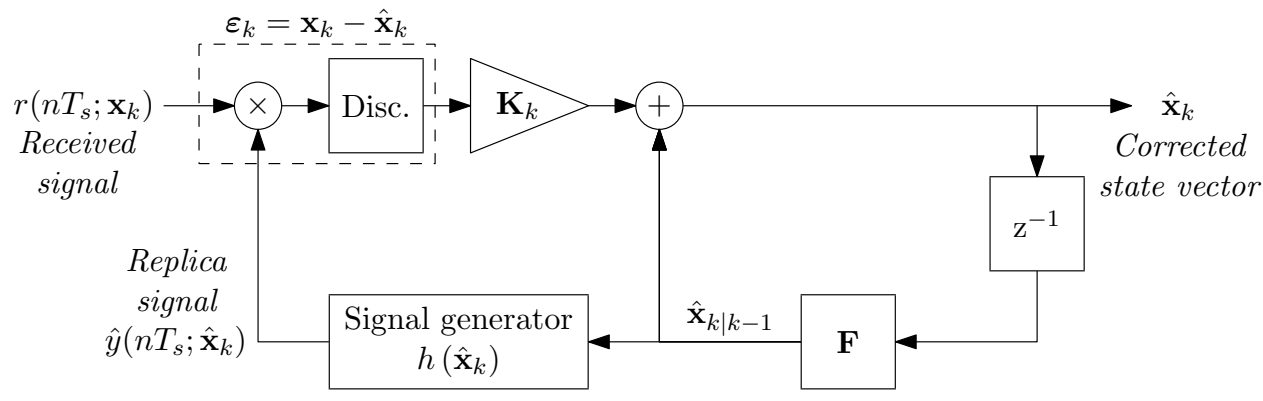

Fig. 1. Kalman filter-based tracking diagram.

to $6 \mathrm{~dB}$ (with respect to the Costas discriminators) since they track the full four quadrant range of the input signal [12]. The maximum likelihood (ML) estimator under these conditions is the four-quadrant arctangent or atan2 (in MATLAB notation), but it implies a high computational burden. A popular alternative is to take the imaginary part of the prompt samples normalized by the prompt envelope as a simple approximation. In fact, this discriminator is proved experimentally to slightly outperform the more complex atan2 function [12], especially at low $C / N_{0}$, hence it is used in our proposed implementation.

$$
\varphi_{e, k}=\frac{1}{2 \pi} \frac{\operatorname{Im}\left(Y_{P, k}\right)}{\left|Y_{P, k}\right|}
$$

Nevertheless, if the navigation message is not available, a Costas discriminator has to be used. In this case, the two-quadrant arctangent discriminator is chosen because it is optimal (ML estimator) at high and low $C / N_{0}$. The main drawback is the high computational burden produced by the non-linear operation. However, it is approximately linear for phase errors near zero, and it is usually implemented using look-up tables.

$$
\varphi_{e, k}=\frac{1}{2 \pi} \operatorname{atan}\left(\frac{\operatorname{Im}\left(Y_{P, k}\right)}{\operatorname{Re}\left(Y_{P, k}\right)}\right)
$$

The noise that corrupts the measurements has also to be included into the model. This is achieved by designing the measurement noise covariance matrix $\mathbf{R}_{k}$. Since the carrier and code measurements are uncorrelated, $\mathbf{R}_{k}$ results in the following diagonal matrix:

$$
\mathbf{R}_{k}=\left[\begin{array}{cc}
\sigma_{\tau_{e, k}}^{2} & 0 \\
0 & \sigma_{\phi_{e, k}}^{2}
\end{array}\right]
$$

where $\sigma_{\tau_{e, k}}^{2}$ and $\sigma_{\phi_{e, k}}^{2}$ are the code and carrier measurement variances, respectively. The computation of open-loop discriminator variances can be obtained either with approximated expressions [5], [11], or by prior Monte-Carlo simulations.

Finally, it should be considered that the normalized carrier states may exceed the normalized $\left[-\frac{1}{2}, \frac{1}{2}\right]$ range after performing any addition or subtraction, which may produce an error on the estimation. Therefore, a wrapping operation must be applied.

\section{E. NCO update}

In the KF structure, the code and carrier numerically controlled oscillators ( $\mathrm{NCO}$ ) are implemented with the transition equation $^{1} \hat{\mathbf{x}}_{k \mid k}=\mathbf{F} \hat{\mathbf{x}}_{k \mid k-1}$. Once the Doppler frequency is estimated, it is used to generate the code and carrier replicas by applying the carrier aiding. In addition, the residual code and carrier phase errors are also used to compensate residual errors, resulting in the phase-and-rate feedback NCO procedure.

\section{High-Sensitivity Kalman Filter-Based TRACKING}

Since the previous architecture may have difficulties to maintain tracking lock at low $C / N_{0}$, efforts have been devoted to design a new tracking architecture capable of being robust to high-sensitivity conditions while still providing a low complexity and good performance. For that purpose, a Kalman filter specialized in high sensitivity is designed. This structure is based on extending the integration time $T$ to compensate the attenuation suffered by the signal under weak signal conditions.

Particularly, the study concentrates on improving the carrier estimation, since it is not as robust as the code estimation in harsh environments. Thus, we focus on the analysis of the phase.

\section{A. Extended correlations}

The concept of extended correlations is introduced here. Basically, the signal $Y_{k}$ obtained after the I\&D block is postprocessed to filter the signal even more. In this case, the postprocessing is based on the coherent or non-coherent accumulation of $1 \mathrm{~ms}$-correlated (for the case of GPS L1) signals. As it is well-known, a non-linear operation is necessary to avoid the data bit transition, if the navigation message is not available. This structure leads to the proposed HS PLL-based discriminator shown in Figure 2, where it can be noticed that the non-coherent accumulation is based on the squaring operation $(\cdot)^{2}$ and not on the squared-modulus operation $|\cdot|^{2}$ of $Y_{k}$, as it would be implemented in a HS DLL-based discriminator.

\footnotetext{
${ }^{1}$ In order to indicate that the state vector $\hat{\mathbf{x}}_{k}$ has indeed been computed with information from the previous sample (i.e. the $(k-1)$-th sample), the double subscript notation $\hat{\mathbf{x}}_{k \mid k-1}$ is herein introduced.
} 


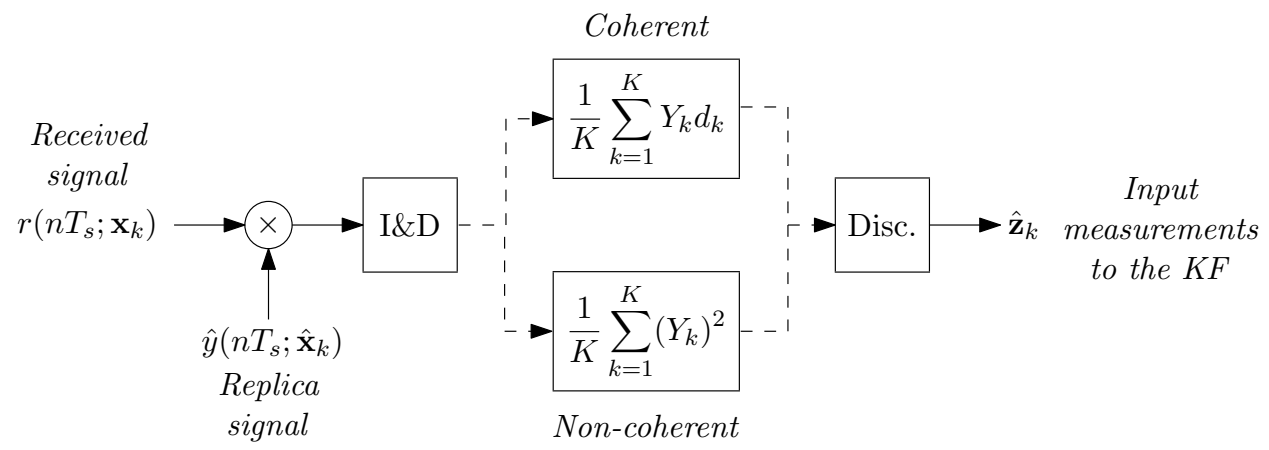

Fig. 2. Comparison between the coherent and non-coherent integration for the HS PLL-based discriminator.

1) Coherent integration: For the case of GPS L1 signals, they are data modulated by a 50 bps BPSK, and thus the integration cannot be extended beyond the $20 \mathrm{~ms}$ bit period. If the navigation message is available, then the bit signs can be compensated, and hence the integration time can be further extended allowing the use of pure PLL discriminators. This assumption is reasonable in receivers that start in good conditions, then the coherent integration can be expressed as

$$
Y_{\text {coh }}^{P L L}=\frac{1}{K} \sum_{k=1}^{K} Y_{k} e^{j \pi b_{k \bmod 20}}
$$

where $K$ is the number of correlated signals over $T$ intervals, and $b_{k \text { mod } 20}$ takes 0 and 1 values due to the bit sign. The correlation interval $T$ is defined to $1 \mathrm{~ms}$, hence the number $K$ will be limited to 20 if the bit information is not available.

Performing some further calculations, (19) can be approximated as

$$
Y_{c o h}^{P L L} \approx A_{c o h} \frac{\sin \left(\pi f_{e} T_{c o h}\right)}{\pi f_{e} T_{c o h}} e^{j\left(\pi f_{e} T_{c o h}+\phi_{e}\right)}+\eta_{c o h},
$$

where $A_{c o h}$ is the resulting signal amplitude after the coherent integration, $T_{c o h}$ is equal to $K T$, and $\eta_{c o h}$ is the filtered Gaussian noise. Since the noise is preserved zeromean Gaussian, it tends to zero as the integration increases. Thus, the coherent amplitude obtained can be defined as $A_{\text {coh }}=R\left(\tau_{e}\right) \sqrt{C / N_{0} T_{\text {coh }}}$, assuming the noise variance normalized to 1 .

Despite the noise reduction, the coherent integration cannot be extended infinitely. Since the integration is a low-pass filter (LPF), its bandwidth $B_{L P F}$, which is equal to $1 / T_{c o h}$, has to be sufficiently large to leave the signal components undistorted. In other words, the rate of change of the signal or signal dynamics, i.e. the Doppler frequency error, has to be confined inside the filter bandwidth because it attenuates the signal by the $\operatorname{sinc}\left(f_{e} T_{c o h}\right)$ factor. This leads to a trade-off between noise reduction and dynamics response.

Additionally, the filtered signal after integration has a biased carrier with respect to the expected argument $2 \pi f_{e} T_{c o h}+\phi_{e}$. This is because the argument of this filtered signal is

$$
\arg \left\{\frac{1}{K} \sum_{k=1}^{K} Y_{k} e^{j \pi b_{k \bmod 20}}\right\}=\pi f_{e} T_{c o h}+\phi_{e}+\eta_{c o h, \phi}
$$

and thus the estimation of the state vector may not be corrected properly. As it can be seen in (21), the phase obtained is the average over the interval $T_{c o h}$ plus phase noise $\left(\eta_{c o h, \phi}\right)$. A solution for this error is to adjust the Kalman filter to be sufficiently reactive to correct it. In this case, the estimations of the KF may oscillate considerably due to the signal dynamics. This oscillation has to be controlled by the designer in order to avoid instabilities.

2) Non-coherent integration: The first measure to overcome the data bit is the use of Costas discriminators. These discriminators are not sensitive to the bit sign, and hence they can operate without compensating the navigation message. Moreover, if the bit synchronisation is assumed, the optimal pre-detection integration can be achieved using a coherent integration of $20 \mathrm{~ms}$. Although this integration obtains a gain of $13 \mathrm{~dB}$ with respect to an integration of $1 \mathrm{~ms}$, the SNR is not sufficiently increased to detect the signal in high-sensitivity conditions.

Alternatively, the extension of the integration time can be achieved by applying a non-coherent process. The most popular approach is the operation of squaring the signal before integration. Thus, for the case of GPS L1, 20 ms-coherent integrations are squared and accumulated. Therefore, the noncoherent and coherent combined integration is expressed as

$$
Y_{n c o h}^{P L L}=\frac{1}{M} \sum_{m=1}^{M}\left(Y_{c o h, m}\right)^{2},
$$

which results in the following

$$
Y_{n c o h}^{P L L} \approx A_{n c o h} \frac{\sin \left(2 \pi f_{e} T_{n c o h}\right)}{2 \pi f_{e} T_{n c o h}} e^{j\left(2 \pi f_{e} T_{n c o h}+2 \phi_{e}\right)}+\eta_{n c o h},
$$

where $A_{n c o h}$ is equal to $A_{c o h}^{2} R\left(\tau_{e}\right)^{2}\left(\operatorname{sinc}\left(f_{e} T_{c o h}\right)\right)^{2}, T_{n c o h}$ is equal to $M T_{\text {coh }}$, and $\eta_{n c o h}$ denotes the filtered noise after the non-coherent integration. Since $M K T \gg K T$ is assumed, the main attenuation factor is the term $\operatorname{sinc}\left(2 f_{e} T_{c o h}\right)$. This non-coherent loss shows that the effect of the dynamics have been doubled due to the squaring operation. Therefore, the dynamics tracking ability is halved with respect to the coherent approach for the same integration period $\left(T_{c o h}=T_{n c o h}\right)$.

Despite of the bit removal, the use of pure PLL discriminators is still possible. Using a four-quadrant arctangent or an 
imaginary part normalized discriminator, the argument of the non-coherent integration is

$\arg \left\{\frac{1}{M} \sum_{m=1}^{M}\left(\frac{1}{K} \sum_{k=1}^{K} Y_{k}\right)^{2}\right\}=2 \pi f_{e} T_{n c o h}+2 \phi_{e}+\eta_{n c o h, \phi}$.

From the above expression, the detected argument can be seen as twice the average of the true phase error over the interval $T_{n c o h}$. Therefore, if the carrier phase error $\phi_{e}$ has been correctly estimated and compensated in the previous intervals, the systematic error of the estimation is removed.

3) IQ integration: An alternative solution to the only use of the squaring operation, which can amplify the noise, is found by developing the classic $I Q$ discriminator. The proposed noncoherent approach is expressed as

$$
Y_{n c o h}^{P L L}=\frac{\sum_{m=1}^{M} \operatorname{Im}\left(Y_{P, k}^{2}\right)}{\sum_{m=1}^{M}\left|Y_{P, k}\right|^{2}} .
$$

Experimentally, it has been found that the $I Q$ integration approximates the classical approaches (coherent or noncoherent). However, when the input phase is bigger than 0.1 cycles approximately, the $I Q$ integration diverges from the result obtained with the classical approaches.

\section{B. System dynamic model}

Once the HS discriminators have been presented, the Kalman filter is applied to complete the signal tracking architecture, as is shown in Figure 3. The KF required maintains the structure described in Section III, but some modifications are necessary to consider the effect of the integration time extension prior to the discriminator.

When the integration interval is extended $K$ times, the incoming measurements are decimated reducing its incoming rate to $1 / K T$. During this period, the measurements of the $\mathrm{KF}$ are disconnected, which avoids the correction of the parameters estimates.

The projection of the states, which operates as the code and carrier NCO, is not affected by the extension of the integration time since it is updated each correlation $k$. Nevertheless, the decimation produced by the integration causes a discontinuity on the predicted Kalman gains. Using the common expression in Kalman theory for the prediction of the state vector covariance matrix $\mathbf{P}_{k+1 \mid k}$,

$$
\mathbf{P}_{k+1 \mid k}=\mathbf{F P}_{k \mid k} \mathbf{F}^{T}+\mathbf{Q},
$$

it can be noticed that the current matrix $\mathbf{P}_{k \mid k}$ is not properly projected. The interval between the current measurement and the following is no longer 1 sample, but $K$ samples. Thus, (26) can be rewritten as

$$
\mathbf{P}_{k+K \mid k}=\mathbf{F}^{K} \mathbf{P}_{k \mid k}\left(\mathbf{F}^{K}\right)^{T}+\sum_{n=0}^{K-1} \mathbf{F}^{n} \mathbf{Q}\left(\mathbf{F}^{n}\right)^{T}
$$

where the resulting transition matrix considers the effect of the integration since

$$
\mathbf{F}^{K}=\left[\begin{array}{cccc}
1 & 0 & \beta & \frac{\beta}{2} \\
0 & 1 & 1 & \frac{1}{2} \\
0 & 0 & 1 & 1 \\
0 & 0 & 0 & 1
\end{array}\right]=\left[\begin{array}{cccc}
1 & 0 & \beta K & \frac{\beta}{2} K^{2} \\
0 & 1 & K & \frac{1}{2} K^{2} \\
0 & 0 & 1 & K \\
0 & 0 & 0 & 1
\end{array}\right]
$$

This new formulation adapts the Kalman gains to the integration specified. The extension of the integration increases the update interval, consequently the residual error between the estimation and the true value increases during the integration interval. Thus, the increased error is taken into account by applying higher Kalman gains.

Another important issue about the system dynamic model is concerned with the state transition noise covariance matrix Q. This matrix tries to model the error produced by the non-deterministic dynamic processes, as it was shown in Section III. Here, the matrix $\mathbf{Q}$ is tuned for the worst-case scenario, which is determined by the dynamics during the integration interval. Although the performance at high $C / N_{0}$ may decrease, this procedure eases the implementation and testing of the tracking architecture.

\section{Measurement noise}

The noise of the measurements is also an important information to include into the KF. Depending on this information, the $\mathrm{KF}$ will increase or decrease the weight (i.e. Kalman gains) applied to each measurement. If the measurements are very corrupted by noise, the Kalman gains should be very small. Nevertheless, the extended correlations introduced improve the quality of the measurements, thus the measurement noise covariance matrix $\mathbf{R}_{k}$ introduced in Section III has to be adapted to the integration interval. Following the approximations described by [11], the open-loop discriminator variances for the DLL $\sigma_{\tau_{e, k}}^{2}$ and the PLL $\sigma_{\phi_{e, k}}^{2}$, expressed as

$$
\begin{aligned}
\sigma_{\tau_{e, k}}^{2} & =\frac{1}{\left(C / N_{0}\right)_{k} T}\left(\Delta+\frac{\Delta}{1-\Delta} \frac{1}{2\left(C / N_{0}\right)_{k} T}\right) \\
\sigma_{\phi_{e, k}}^{2} & =\frac{1}{8 \pi^{2}\left(C / N_{0}\right)_{k} T}\left(1+\frac{1}{2\left(C / N_{0}\right)_{k} T}\right)
\end{aligned}
$$

can be modified according to the $C / N_{0}$ at time $t_{k}$ and the extended correlation $T$ designed. For instance, the $C / N_{0}$ can be detected with the widely known Narrow-Wideband Power Ratio (NWPR) estimator.

\section{Performance eVAluation}

\section{A. Theoretical performance}

The theoretical performance of the proposed architecture can be assessed by computing the phase variance as function of the $C / N_{0}$ and analysing a 200 ms-extended integration. Moreover, the lower and upper performance bound are defined by the normalized modified Cramer-Rao bound (MCRB) for 


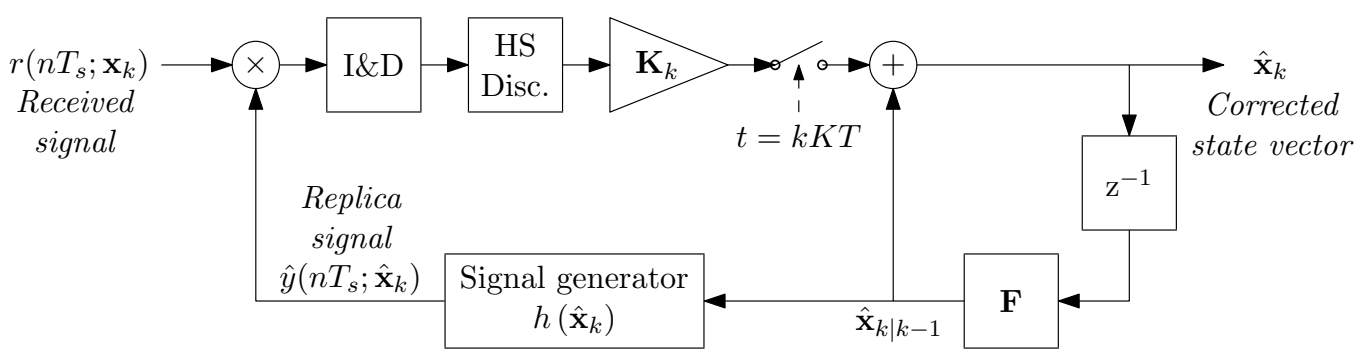

Fig. 3. Kalman filter-based tracking diagram with extended integrations.

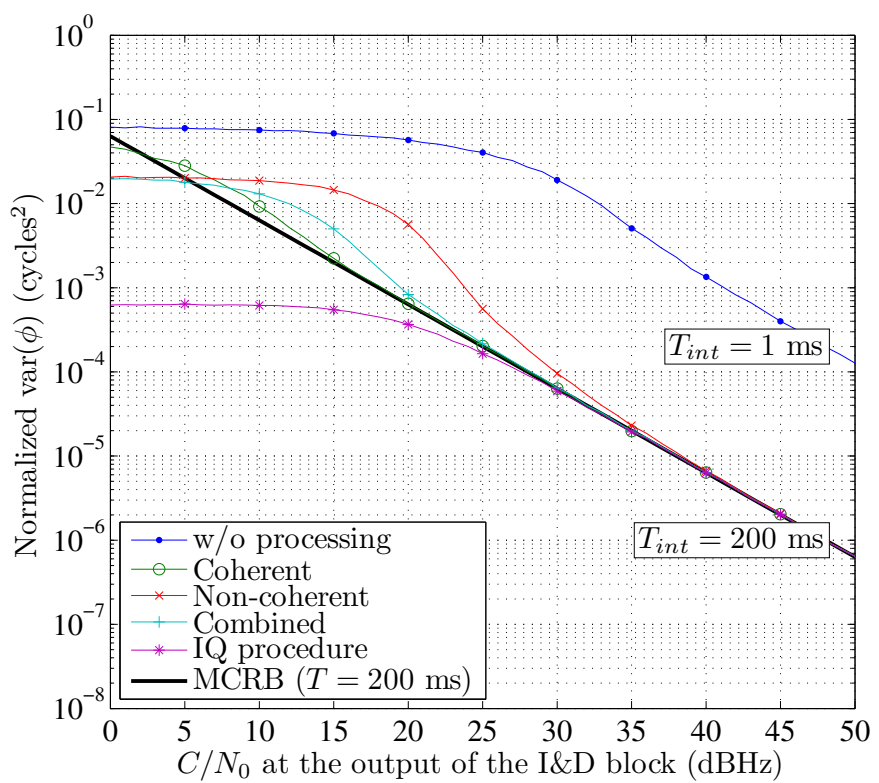

Fig. 4. Kalman filter-based tracking diagram with extended integrations.

phase estimation and the phase detected without any processing after the I\&D block, respectively. The normalized MCRB is given by [13]

$$
M C R B_{\phi}=\frac{1}{2 C / N_{0} T} \cdot \frac{1}{4 \pi^{2} L} .
$$

The results of this comparison are presented in Figure 4. Clearly, the extension of the integration time improves the resulting phase variance. But, only the coherent integration is nearly optimal at low $C / N_{0}$. As it can be seen, the fully noncoherent method, which squares 1 ms-correlated outputs, has the worst performance, nevertheless it is improved by combining non-coherent integrations of $20 \mathrm{~ms}$-coherent integration. Regarding to the $I Q$ procedure, it is shown that its variance is below the MCRB for low $C / N_{0}$. This is because the arcsine discriminator (required in the $I Q$ procedure) collapses when there is too much noise and the discriminator outputs become random uniformly distributed. This effect can also be noticed in the other methods, but for lower $C / N_{0}$. Since the fourquadrant arctangent (used in the other methods) has double dynamic range with respect to the arcsine, it collapses later.

\section{B. Practical performance}

In order to assess the practical performance, the tracking architecture is implemented on a software GPS receiver. Nevertheless, the evaluation of the signal tracking is not a straightforward task when the receiver is under unknown harsh conditions, for instance when using real signal. In this case, it is difficult to ascertain the correct operation of the signal tracking by means other than just the inference from lock detectors or $C / N_{0}$ estimators. Moreover, these detectors have a bad performance at low $C / N_{0}$, and consequently their information is not very reliable. Therefore, the most reasonable solution is to track a synthetic signal, as a prior step before testing the system with real signals, which is left as future work.

Using the synthetic signal to simulate a real GPS signal, the validation is simplified to the carrier tracking only, since the code tracking is typically more robust to high-sensitivity scenarios. Thus, it is considered that the code wipe-off is previous to the signal generation, and that the code correlation and the I\&D operation are already done. Moreover, the code delay error $\tau_{e}$ is considered practically zero $\left(R\left(\tau_{e}\right) \approx 1\right)$, due to the robustness of the code and the carrier-aiding application. In addition, since the integration of the I\&D block has a maximum duration of one bit (i.e. $20 \mathrm{~ms}$ ), its averaging effect and its sinc attenuation factor are assumed negligible.

According to the previous considerations, the scenario under test models a vehicle moving with constant velocity in an urban canyon. The receiver is assumed to be initialized in open sky or with good visibility conditions, such as in street intersections, achieving bit synchronization. Ten seconds after its initialization, the $C / N_{0}$ is decreased from $45 \mathrm{dBHz}$ to $15 \mathrm{dBHz}$ in a sudden fall, to address the capabilities of our implementation at weak signal levels. The steady dynamics proposed are determined by the residual Doppler frequency, which is assumed to be a ramp defined by the initial frequency $f_{e}$ and the initial frequency rate or acceleration $\dot{f}_{e}$. The initial frequency is set to only $2 \mathrm{~Hz}$, and the initial frequency rate (i.e. due to the satellite motion) is set to $0.5 \mathrm{~Hz} / \mathrm{s}$.

Once the scenario is specified, the methods proposed are evaluated taking into account two cases. Firstly, the integration time $T_{\text {int }}$ of the I\&D block is set to $20 \mathrm{~ms}$ and no processing is used to extend the correlation beyond the data bit. Secondly, the different methods are introduced to extend the integration until $200 \mathrm{~ms}$. 

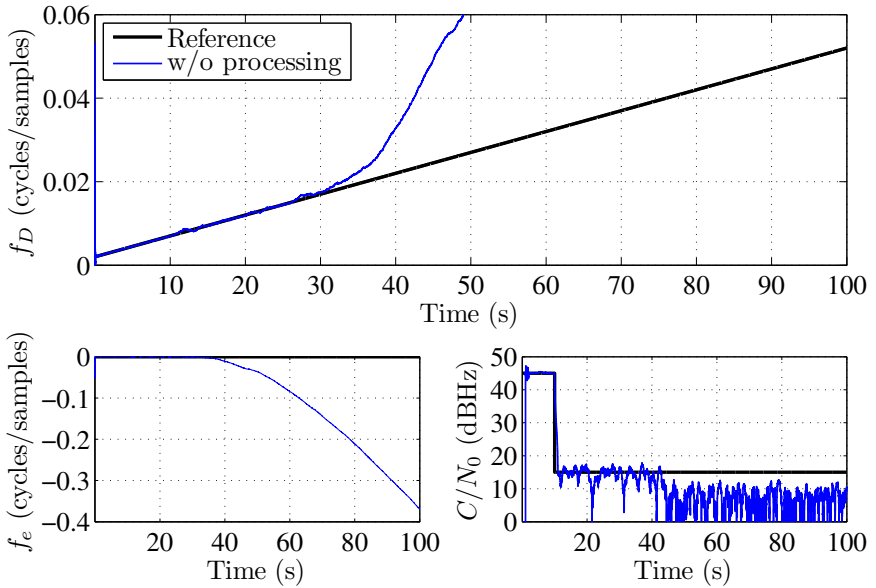

Fig. 5. Normalized frequency (top), normalized frequency error (left) and $C / N_{0}$ estimate (right) without any processing method for $T_{i n t}=20 \mathrm{~ms}$.
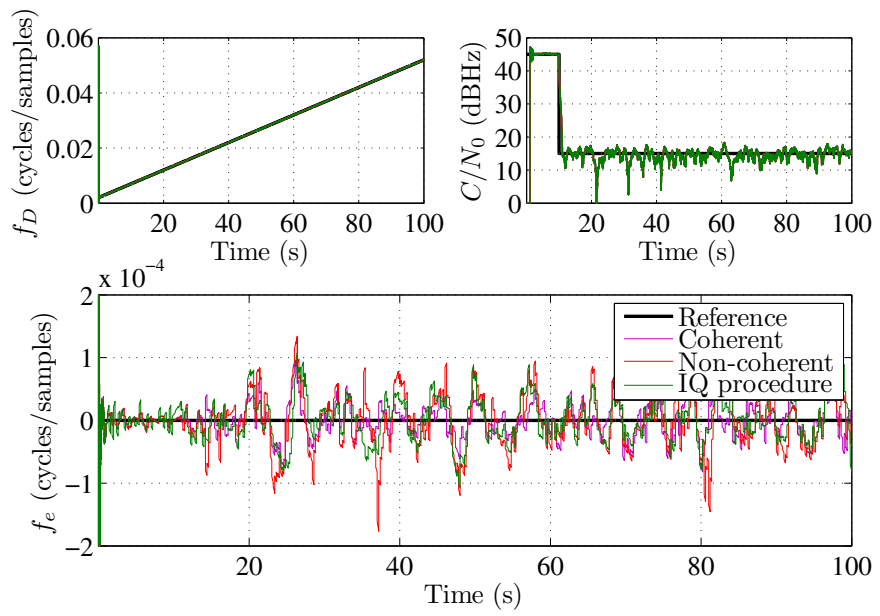

Fig. 6. Normalized frequency (top left), $C / N_{0}$ estimate (top right) and normalized frequency error (bottom) of the methods proposed for $T_{\text {int }}=200$ $\mathrm{ms}$. In the first two figures, the difference between plots is not noticeable.

As it can be seen in Figure 5, the signal tracking cannot be maintained at low $C / N_{0}$ when no processing technique is used. Particularly, it loses lock near 40 seconds, as it can be assessed from the frequency error and the $C / N_{0}$ estimate. On the contrary, when HS processing techniques are introduced, the signal can be tracked with a remarkable accuracy, as it is shown in Figure 6.

The results presented show the necessity to introduce extended correlations in order to operate under weak signal conditions. However, this signal processing is not the only responsible for the high-sensitivity achievement. The configuration of the Kalman filter is also critical for our purpose. Depending on the knowledge of the system dynamic model, the tracking threshold can be improved or not. In our case, a conservative configuration has been set weighting more the dynamic model in order to reduce the impact of the noisy measurements. For that reason, the three methods compared in Figure 6 show similar perfomances.
Nevertheless, the system dynamic model is usually not completely known, for instance when the vehicle dynamics are not constant. In these cases, the measurements have to be considered in order to properly correct the parameter estimations. Under these changing conditions, the use of the presented extended correlations may reduce the dynamics response. As it was introduced in Section IV, there is a tradeoff between the noise reduction and the dynamics supported. Other approaches such as the adoption of an FLL-based KF may help to cope with the user's dynamics in more effective way than a PLL-based KF.

\section{CONCLUSION}

A new Kalman filter-based architecture for robust and highsensitivity tracking in GNSS receivers has been presented. The proposed idea is to combine the low complexity and optimality of the Kalman filter with a signal processing of the measurements. This processing is based on implementing extended correlations. For its implementation, different methods have been analysed in order to track signals with a standalone architecture under harsh conditions. The results have shown that the proposed architecture is able to track signals with steady dynamics at a $C / N_{0}$ as low as $15 \mathrm{dBHz}$. It has been noticed that the new KF formulation improves the robustness of the integration methods presented. Its application to real signals is left as future work, as well as, its improvement in changing dynamics.

\section{REFERENCES}

[1] M. Psiaki and H. Jung, "Extended Kalman filter methods for tracking weak GPS signals," in Proc. of ION GPS 2002, 9-12 Sept. 2002.

[2] N. Ziedan and J. Garrison, "Extended Kalman Filter-Based Tracking of Weak GPS Signals under High Dynamic Conditions," in Proc. of ION GNSS 2004, 21-24 Sept. 2004.

[3] C. O'Driscoll and G. Lachapelle, "Comparison of Traditional and Kalman Filter Based Tracking Architectures," in Proc. of European Navigation Conference 2009 (ENC09), 3-6 May 2009.

[4] J.-H. Won, D. Dötterböck, and B. Eissfeller, "Performance Comparison of Different Forms of Kalman Filter Approach for a Vector-Based GNSS Signal Tracking Loop," in Proc. of ION GNSS 2009, 22-25 Sept. 2009.

[5] A. Van Dierendonck, "GPS Receivers," in Global Positioning System: Theory and Applications, B. Parkinson and J. Spilker, Eds. Washington: AIAA Inc., 1996, vol. 1, ch. 8, pp. 329-405.

[6] D. Polk and S. Gupta, "Quasi-optimum digital phase-locked loops," IEEE Transactions on Communications, vol. 21, no. 1, pp. 75-82, 1973.

[7] A. Patapoutian, "On phase-locked loops and Kalman filters," IEEE Transactions on Communications, vol. 47, no. 5, pp. 670-672, 1999.

[8] K. Kaindl, "Optimal GPS-Tracking Even in Case of Line-of-Sight Loss," in Proc. of ION GPS/GNSS 2003, 9-12 Sept. 2003.

[9] T. Pany, R. Kaniuth, and B. Eissfeller, "Deep Integration of Navigation Solution and Signal Processing," in Proc. of ION GNSS 2005, 13-16 Sept. 2005.

[10] M. Lashley, D. M. Bevly, and J. Y. Hung, "Performance Analysis of Vector Tracking Algorithms for Weak GPS Signals in High Dynamics," IEEE Journal of Selected Topics in Signal Processing, vol. 3, no. 4, pp. 661-673, Aug. 2009.

[11] C. Gernot, "Development of Combined GPS L1/L2C Acquisition and Tracking Methods for Weak Signals Environments," PhD Thesis, Dept. of Geomatics Eng., University of Calgary, 2009, UCGE Report 20296.

[12] B. J. Ward, P.W. and C. Hegarty, "Satellite Signal Acquisition, Tracking, and Data Demodulation," in Understanding GPS: Principles and Applications, 2nd ed., E. Kaplan and C. Hegarty, Eds. Boston, London: Artech House Publishers, 2006, ch. 5, pp. 153-241.

[13] U. Mengali and A. D'Andrea, Synchronization techniques for digital receivers. New York: Plenum, 1997. 